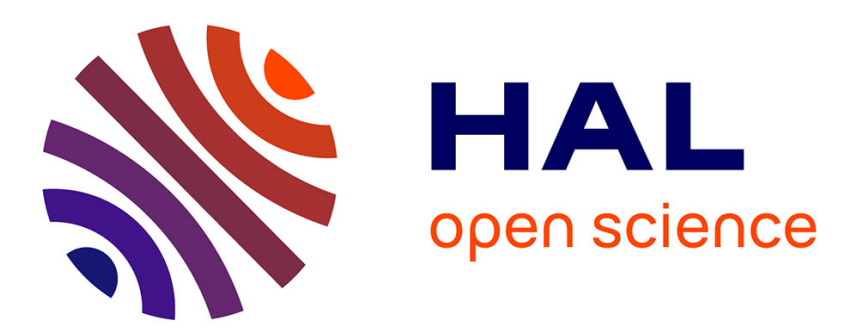

\title{
Les arrangements internationaux pour garantir les Etats non dotés d'armes nucléaires contre l'emploi ou la menace de ces armes
}

\author{
Abdelwahab Biad
}

\section{- To cite this version:}

Abdelwahab Biad. Les arrangements internationaux pour garantir les Etats non dotés d'armes nucléaires contre l'emploi ou la menace de ces armes. Annuaire Français de Droit International, 1997, 10.3406/afdi.1997.3446 . hal-02641604

\section{HAL Id: hal-02641604 \\ https://hal-normandie-univ.archives-ouvertes.fr/hal-02641604}

Submitted on 28 May 2020

HAL is a multi-disciplinary open access archive for the deposit and dissemination of scientific research documents, whether they are published or not. The documents may come from teaching and research institutions in France or abroad, or from public or private research centers.
L'archive ouverte pluridisciplinaire HAL, est destinée au dépôt et à la diffusion de documents scientifiques de niveau recherche, publiés ou non, émanant des établissements d'enseignement et de recherche français ou étrangers, des laboratoires publics ou privés. 


\section{Les arrangements internationaux pour garantir les Etats non dotés} d'armes nucléaires contre l'emploi ou la menace de ces armes

M. Abdelwahab Biad

\section{Citer ce document / Cite this document :}

Biad Abdelwahab. Les arrangements internationaux pour garantir les Etats non dotés d'armes nucléaires contre l'emploi ou la menace de ces armes. In: Annuaire français de droit international, volume 43, 1997. pp. 227-252;

doi : 10.3406/afdi.1997.3446

http://www.persee.fr/doc/afdi_0066-3085_1997_num_43_1_3446

Document généré le 12/03/2016 


\title{
LES ARRANGEMENTS INTERNATIONAUX POUR GARANTIR LES ÉTATS NON DOTÉS D'ARMES NUCLÉAIRES CONTRE L'EMPLOI OU LA MENACE DE CES ARMES
}

\author{
ABDELWAHAB BIAD
}

\section{INTRODUCTION}

Les essais nucléaires déclenchés par l'Inde puis en réaction par le Pakistan en mai 1998 viennent remettre au devant de l'actualité la question de la menace nucléaire. Ces deux frères ennemis qui n'ont souscrit à aucun engagement international de non-prolifération viennent de forcer l'entrée du Club atomique invoquant les mêmes arguments de sécurité que ceux utilisés en leur temps par les puissances nucléaires pour justifier leur arsenal atomique. Ces deux pays estiment faire l'objet d'une menace nucléaire implicite (chinoise pour l'Inde et indienne pour le Pakistan). Le seul mérite des Indiens et des Pakistanais dans cette affaire est d'avoir posé le véritable problème - l'absence de garanties de sécurité universelles prohibant l'emploi d'armes nucléaires contre des États qui en sont dépourvus - tout en proposant une solution inadéquate, la nucléarisation.

L'avis consultatif de la CIJ du 8 juillet 1996 sur la "licéité de la menace ou de l'emploi d'armes nucléaires" avait déjà été l'occasion d'un débat révélant l'intérêt que la question du recours à cette catégorie d'arme de destruction massive soulève dans la communauté internationale à l'ère post-guerre froide (1).

Il ne s'agit évidemment pas ici de s'interroger sur la licéité de l'emploi de l'arme nucléaire mais de s'intéresser au non-emploi sélectif, à l'égard d'une catégorie d'États, les États non dotés de l'arme nucléaire (ENDAN). A ce propos, l'Assemblée générale des Nations Unies qui est à l'origine de la requête auprès de la CIJ, considère dans les résolutions qu'elle adopte depuis vingt ans sur la question (2), que si la garantie la plus efficace contre l'emploi de l'arme nucléaire est le désarmement nucléaire, en attendant, les

(*) Abdelwahab Biad a été Maître de conférence à l'Institut de Droit de l'Université de Annaba, il enseigne actuellement à la Faculté de Droit des Sciences Économiques et de Gestion de l'Université de Rouen.

$N B$ : L'auteur tient à remercier M. Jean-Michel Favre du ministère des Affaires étrangères pour son aide.

(1) Voir les nombreuses réactions qu'a suscitées cet avis parmi les juristes notamment dans l'AFDI 1996 (contributions de Marc PERrin de Brichambaut, pp. 316-336 et de Vincent CoussiratCoustère, pp. 337-356), ainsi que dans le numéro spécial de la Revue internationale de la Croix-Rouge, janvier-février 1997, $\mathrm{n}^{\circ} 823$ (notamment Luigi Condorelli et Éric David).

(2) Le premier appel en ce sens fut lancé par l'Assemblée à l'occasion de sa première session spéciale consacrée au désarmement (1978), S-10/2, § 59 . 
États dotés d'armes nucléaires (ÉDAN) devraient donner des assurances aux ENDAN contre le recours ou la menace du recours à ces armes. Cette revendication qui fait l'objet d'un examen régulier par l'Assemblée générale et la Conférence du désarmement de Genève est perçue comme légitime, y compris par les puissances nucléaires, ces assurances de sécurité représenteraient la juste contrepartie à la renonciation à l'option nucléaire par de nombreux Etats.

En fait cette question peut être appréhendée sous différents aspects. Ces assurances de sécurité peuvent aussi bien viser le non-emploi d'armes nucléaires, c'est à dire les garanties négatives, que l'assistance contre l'emploi que l'on désigne par garanties positives. Les propositions élaborées en ce sens depuis trois décennies présentent la caractéristique d'être extrêmement fragmentées. Elles pourraient être classées en six catégories : prohibition du recours à l'arme nucléaire; non-emploi en premier; renonciation à l'emploi à l'égard des zones exemptes d'armes nucléaires (ZEAN); engagement unilatéral et conditionnel de non-emploi en faveur des ENDAN parties au Traité sur la non-prolifération des armes nucléaires (TNP); non-emploi inconditionnel à l'égard des ENDAN; devoir d'assistance contre l'emploi. A l'exception de la dernière toutes les formules énumérées ici concernent directement ou indirectement les garanties négatives.

Nous verrons à travers les arrangements en vigueur aujourd'hui les solutions très diversifiées et souvent contestées qui ont été apportées dans ce domaine. Ces solutions sont perfectibles mais les efforts actuelles visant à élaborer une formule commune sur les garanties négatives se heurtent à des difficultés considérables.

\section{I. - LES ARRANGEMENTS EN VIGUEUR}

Les arrangements internationaux en vue de garantir les ENDAN contre l'emploi ou la menace d'emploi d'armes nucléaires seraient tout à fait conformes aux dispositions de la Charte des Nations Unies et en particulier aux articles 1 et 2 . En effet, l'article 1 fait référence à l'engagement des États à "prendre des mesures collectives efficaces en vue de prévenir et d'écarter les menaces à la paix". D'autre part, l'article $2 \S 4$ pose que les États "s'abstiennent dans leurs relations internationales, de recourir à la menace ou à l'emploi de la force, soit contre l'intégrité territoriale ou l'indépendance politique de tout État". Ainsi, la question des garanties négatives peut être envisagée à la fois dans le cadre des mesures de prévention des conflits aussi bien que dans le contexte de la limitation du recours à la force. S'il ne fait aucun doute que ces arrangements confortent la paix et la sécurité internationale, la question cruciale est comment les envisager.

Et d'abord qu'est ce qu'on entend par arrangements internationaux efficaces? L'expression arrangements internationaux généralement utilisée pour désigner les garanties négatives de sécurité fait aujourd'hui consensus parce que elle est suffisamment floue pour laisser à chaque État l'espoir que l'arrangement auquel il pense n'est pas à priori exclu. A ce propos, les réalisations et les propositions actuelles portent sur un large éventail d'arrangements allant de la déclaration unilatérale à la convention internationale en passant par une résolution du Conseil de sécurité. L'imprécision du terme 
arrangement révèle donc l'ampleur des divergences qui opposent actuellement les États sur la question du non-emploi de l'arme nucléaire contre les ENDAN. Ces divergences n'ont toutefois pas paralysé les efforts internationaux qui se sont traduits par des réalisations certes modestes mais qui ont le mérite d'exister.

Les arrangements en vigueur aujourd'hui se caractérisent par une extrême diversité tant dans la forme juridique que dans le contenu de l'engagement. Ils se sont traduits concrètement par une renonciation conditionnelle à l'emploi formulée dans des déclaration unilatérales, mais aussi par l'interdiction du recours à l'arme nucléaire énoncée dans le cadre des traités instituant des ZEAN, et enfin par le devoir à l'assistance contre l'emploi contenu dans des résolutions du Conseil de sécurité.

\section{A. Les déclarations unilatérales des ÉDAN}

A l'occasion de la première session spéciale de l'Assemblée générale des Nations Unies consacrée au désarmement (1978), les ÉDAN avaient formulé des déclarations unilatérales en vertu desquelles ils renonçaient sous certaines conditions à l'emploi ou à la menace de l'emploi d'armes nucléaires contre des ENDAN. Aujourd'hui les déclarations des cinq ÉDAN sur les garanties de sécurité sont, à l'exception du cas de la Chine, pratiquement identiques depuis leur reformulation et leur harmonisation en avril 1995 dans le cadre de la résolution 984 du Conseil de sécurité (3). Ainsi, les États-Unis, le Royaume-Uni, la France et la Russie se sont engagés dans des déclarations séparées à ne pas employer d'armes nucléaires contre des États qui n'en sont pas dotés et qui sont parties au TNP, sauf dans le cas d'une invasion ou de toute autre attaque contre elles ou leurs alliés en alliance ou en association avec un ÉDAN. Les États-Unis (4) et le RoyaumeUni (5) ont fait preuve de la plus grande constance dans la formulation de leurs garanties négatives qui n'a pratiquement pas évolué depuis 1978. L'harmonisation des garanties réalisée en 1995 est en fait un alignement de la France et de la Russie sur la position des deux puissances anglo-saxonnes.

Les positions de ces deux pays sur les garanties de sécurité ont en effet, connu une évolution notable. La France s'était en 1978 engagée à ne pas utiliser d'armes nucléaires contre les États appartenant aux ZEAN sauf en cas de légitime défense (6), mais en 1982, Paris a reformulé sa position en indiquant qu'elle n'utiliserait pas d'armes nucléaires contre un ENDAN «qui se serait engagé à le demeurer» (7). Depuis son adhésion au TNP, elle mentionne explicitement les ENDAN parties à ce Traité comme les bénéficiaires des garanties (8). La Russie a également reformulé à partir de 1993 les garanties négatives données en 1978 puis en 1982 par l'URSS. Il n'est plus question de l'engagement pris en 1982 "de ne pas utiliser en premier l'arme nucléaire»(9). La nouvelle déclaration russe stipule qu'elle s'appuie

(3) La résolution 984 (1995) adoptée le 11 avril 1995 "Prend acte avec satisfaction" des déclaration des ÉDAN sur les garanties de sécurité.

(4) Déclaration des États-Unis à la Conférence du désarmement, CD/1305.

(5) Déclaration du Royaume-Uni à la Conférence du désarmement, CD/1306.

(6) A/S-10/PV.3 (25 mai 1978).

(7) A/S-12/PV 9, p. 69, (11 juin 1982).

(8) Déclaration de la France à la Conférence du désarmement, CD/1307.

(9) A/S-12/PV.12, pp. 22-25 (15 juin 1982). 
sur "les dispositions du document établissant la doctrine militaire de la Russie»(10). Cette position constitue une rupture avec l'activisme soviétique en matière de désarmement. La Russie s'est ainsi rapprochée de la position plus classique des puissances occidentales et n'accorde désormais des garanties négatives qu'aux ENDAN parties au TNP sauf en cas d'attaque contre elle et ses alliés en alliance avec une puissance nucléaire.

La Chine a pour sa part réaffirmé l'engagement «de ne jamais, et en aucune circonstance employer ou menacer d'employer des armes nucléaires contre des États qui n'en sont pas dotés ou des zones qui en sont exemptes». Cette renonciation inconditionnelle formulée dès 1964 au moment de son entrée dans le club atomique a été renouvelée en 1978 et en 1982 (11). Elle a précisé en 1995 que cet engagement "s'applique naturellement" aux ENDAN parties au TNP ainsi qu'aux États "qui ont pris des engagements internationalement contraignants comparables par lesquels ils s'interdisaient de fabriquer ou d'acquérir des dispositifs explosifs nucléaires" (12). Il s'agit ici des Etats situés dans des ZEAN. La déclarations chinoise est la seule qui inclue aussi la renonciation à la menace d'employer des armes nucléaires. L'omission volontaire de ce terme par les autres puissances présenterait l'avantage d'assurer une marge de manœuvre dans des situations de crise ou le chantage nucléaire pourrait s'avérer fructueux.

Pour les ÉDAN ces engagements constituent des assurances de sécurité fermes, crédibles et fiables en faveur des ENDAN (13). Les pays non-alignés (PNA) membres du Groupe des 21, ne partagent pas ce point de vue, estimant qu'a l'exclusion du cas de la Chine les déclarations unilatérales des ÉDAN s'appuyaient sur des considérations stratégiques propres à ces puissances et ne pouvaient de ce fait répondre au souci légitime de sécurité des États qui ont renoncé à l'option nucléaire (14). Ils déplorent que ces déclarations présentent l'inconvénient d'être assorties d'exceptions qui affaiblissent la portée et la validité de l'engagement qu'elles visent.

Quelle est la validité de tels engagements? On est ici en présence d'actes unilatéraux étatiques portant renonciation sous certaines conditions à l'emploi ou à la menace d'emploi de l'arme nucléaire. Il ne fait aucun doute qu'il s'agit ici d'une manifestation de volonté non équivoque créant une obligation de comportement (15). L'engagement contenu dans ces déclarations n'en est pas moins réversible. Les Etats garants pourraient à tout moment renoncer à l'obligation qui les lie par l'acte unilatéral en invoquant des "circonstances exceptionnelles". Mais, la remise en cause ou la renonciation à l'engagement défini dans l'acte unilatéral pourrait avoir pour conséquence de poser la question de la mise en jeu de la responsabilité de l'auteur, cet engagement ayant entre temps produit des droits pour des États tiers. D'où l'importance ici de la question de la bonne foi, principe essentiel du droit international en matière de respect des obligations.

(10) Déclaration de la Russie à la Conférence du désarmement, CD/1311.

(11) A/S-12/11 (4 mai 1982).

(12) Déclaration de la Chine à la Conférence du désarmement CD/1309.

(13) $\mathrm{CD} / 825$, p. 219.

(14) $\mathrm{CD} / 825$, p. 217 . Le Groupe des 21 rassemble les pays non-alignés et neutres membres de la Conférence du désarmement : Algérie, Argentine, Brésil, Congo démocratique, Cuba, Égypte, Éthiopie, Inde, Indonésie, Iran, Kenya, Maroc, Myanmar (Birmanie), Mexique, Mongolie, Nigeria, Pakistan, Pérou, Sri Lanka, Suède et Venezuela.

(15) Affaire des essais nucléaires (Australie c./France), Recueil CIJ, 1974, p. 270. 
L'imprécision des termes employés dans ces déclarations doit aussi être soulignée. A ce propos la référence à la menace contre les «intérêts vitaux» - qui ne sont pas spécifiquement définis - comme critère invalidant les garanties négatives contribue à l'ambiguïté des assurances négatives données. Que signifie le terme «toute autre attaque" qui s'ajoute à "invasion" utilisé dans les déclarations américaine, britannique, française et russe? Quel contenu donner aux expressions "en alliance avec" et "en association avec" employées successivement par ces trois pays? Enfin, le fait d'ajouter dans les circonstances excluant les garanties l'attaque contre un "allié " et un "État avec lequel ont été pris des engagements de sécurité" alimente également l'incertitude quant à l'identification de ces deux catégories d'États. A ce propos cette incertitude peut être volontaire lorsque les arrangements de sécurité dont bénéficient certains ENDAN de la part des grandes puissances sont tenus secrets et que les pays concernés entretiennent le doute sur l'existence ou non d'une clause impliquant un parapluie nucléaire (16).

On a vu que quatre des cinq puissances nucléaires ont stipulé que l'engagement de non-emploi cesserait de jouer en cas d'attaque contre ellesmêmes, leurs territoires ou des territoires placés sous leur dépendance, leurs forces armées ou leurs alliés. Il ne s'agit pas ici d'une conditionnalité visant toutes les attaques mais seulement celles qui seraient menées avec l'appui d'une autre puissance nucléaire. Outre le fait qu'elle est conforme au droit à la légitime défense formulé dans l'article 51 de la Charte, cette clause de non attaque viserait selon ses auteurs à couvrir tous les imprévus et donc reviendrait à renforcer la crédibilité des engagements souscrits (17). Le Groupe des 21 s'est élevé contre cette position en rejetant l'idée que l'article 51 soit interprété comme légitimant la menace ou l'emploi d'arme nucléaire dans l'exercice du droit de légitime défense en cas d'attaque par des moyens non nucléaires (18). Sur cette question délicate, la CIJ n'avait pu conclure «de façon définitive que la menace ou l'emploi d'armes nucléaires serait licite ou illicite dans une circonstance extrême de légitime défense dans laquelle la survie même de l'État serait en cause" (19). Peut-on considérer qu'une attaque par des moyens conventionnels constitue une "circonstance extrême" mettant en jeu la "survie» de l'État? Mais, on pourrait penser que si le droit international reconnaît le droit naturel de légitime défense individuelle ou collective, en cas d'agression armée, ce droit conformément à une règle coutumière est soumis aux conditions de nécessité et de proportionnalité (20). Une riposte nucléaire contre un attaque par des moyens conventionnels serait manifestement disproportionnée. Sa mise en œuvre risquerait de surcroît de violer les règles du jus in bello. L'argument de la légitime défense invoqué pour couvrir l'emploi de l'arme nucléaire pourrait être interprété comme visant à assurer la primauté du jus ad bellum sur le jus in bello (21).

(16) A ce propos Henry Kissinger a estimé en 1979 que les États-Unis ne prendraient jamais l'initiative d'une attaque nucléaire contre l'URSS pour protéger ses alliés. Il ajouta que "nos alliés européens ne devraient pas continuer de nous demander de multiplier les garanties stratégiques que nous ne pouvons pas donner ou, si nous le pouvons, que nous ne devrions pas honorer, car si nous le faisions, nous risquerions de détruire la civilisation" in : Henry KISSINGER, "NATO defense and the Soviet Threat", Survival (London), novembre-décembre 1979, p. 266.

(17) $\mathrm{CD} / 825$, p. 213 et 219.

(18) CD/1312, voir aussi CD/825, p. 213

(19) Licéité de la menace ou de l'emploi de l'arme nucléaire, §105, 2), E.

(20) Affaire des Activités militaires et paramilitaires au Nicaragua et contre celui-ci (Nicaragua c./Etats-Unis), Recueil CIJ, 1986, p. 94, § 176.

(21) C'est l'opinion exprimée par Henry MEYRowitz, "Le débat sur le non-recours en premier aux armes nucléaires et la déclaration soviétique du 15 juin 1982, AFDI, 1982, pp. 147-167 (cf. p. 161). 
Le principal intérêt des engagements unilatéraux pourrait être en fin de compte de "baliser" le terrain en vue de la négociation ultérieure d'un accord global interdisant l'emploi de l'arme nucléaire contre les ENDAN. Dans ce contexte, les déclarations comporteraient des engagements "transitoires" et "provisoires" en attendant un accord international qui s'en inspirerait tout en les renforçant. Une comparaison entre ces engagements unilatéraux avec les déclarations faites par les puissances nucléaires dans le cadre des accords instituant les ZEAN réserve parfois des surprises.

\section{B. La renonciation à l'emploi dans le contexte des ZEAN}

L'octroi de garanties de non-emploi et non menace de l'emploi d'armes nucléaires contre les ENDAN parties à des ZEAN est considéré comme un facteur essentiel de l'efficacité de ces zones (22). Des garanties négatives sont formulées en termes clairs dans un protocole additionnel au traité instituant la zone, ce protocole est ouvert à la signature et à la ratification par les ÉDAN. Ainsi, s'est constitué un système juridique intéressant associant un double niveau d'engagements contraignants : celui des ENDAN dont le territoire est situé dans la zone couverte par le traité et celui des ÉDAN appelés à ne mener aucune activité susceptible d'affecter le statut d'absence d'armes nucléaires de la zone. Cette formule inaugurée par le Traité sur l'Amérique latine et les Caraïbes (Tlatelolco) a servi de modèle pour les accords sur le Pacifique sud (Rarotonga), l'Afrique (Pelindaba) et l'Asie du sud-est (Bangkok).

Le Traité de Tlatelolco du 14 février 1967 comporte un protocole ouvert aux ÉDAN qui "s'engagent à ne recourir ni à l'emploi d'armes nucléaires ni à la menace de leur emploi contre les parties contractantes au Traité visant l'interdiction des armes nucléaires en Amérique latine" (23). Il faut noter ici que l'engagement de non-emploi s'applique également aux États parties au Traité qui doivent renoncer non seulement à l'essai, à la fabrication, à la production, à l'acquisition, à la réception, à l'entreposage, à l'installation, à la mise en place, à la possession sous quelque forme que ce soit, mais aussi à «l'emploi " d'armes nucléaires (24). On peut s'étonner ici de la mention du non-recours exigé des ENDAN parties (mention que l'on retrouve également dans le traité sur l'Asie du sud-est) qui s'avère superflue pour des pays qui ont renoncé en vertu du traité à acquérir et à posséder des armes nucléaires. Les protocoles pertinents des Traités de Rarotonga (25) (4 août 1985), de Pelindaba (26) (11 avril 1996) et de Bangkok (15 décembre 1995) s'inspirent du modèle de Tlatelolco. Il faut remarquer que pour ce qui concerne l'accord sur l'Asie du sud-est, l'engagement de non-emploi demandé aux puissances nucléaires s'applique non seulement aux États parties au Traité mais aussi "contre la zone exempte d'armes nucléaires d'Asie du sud-est" (27). Cette dernière précision signifierait en pratique qu'un ÉDAN pourrait bénéficier des garanties négatives du fait qu'il est situé dans la zone et sans même être partie au Traité. Cette exigence, jugée inacceptable par la plupart des

(22) Voir Étude complète de la question des zones exemptes d'armes nucléaires sous tous ses aspects, Rapport spécial de la Conférence du Comité du désarmement, CCD/467, 1975, § 115.

(23) Protocole additionnel II, article 3.

(24) Article 1 §a) du Traité.

(25) Protocole 2, article $1^{\text {er }}$.

(26) Protocole I, article $1^{\mathrm{er}}$.

(27) Protocole, article 2. 
ÉDAN, est la principale cause du retard dans la mise en œuvre de cet instrument. Comme pour celui de l'Amérique latine on retrouve également dans ce dernier traité régional, l'exigence faite aux États parties d'Asie du sud-est de ne pas employer d'armes nucléaires à l'intérieur ou à l'extérieur de la zone (28).

En signant et en ratifiant ces protocoles, les ÉDAN ont fait des déclarations interprétatives selon lesquelles ils se réservaient le droit de reconsidérer, dans certaines circonstances, leurs engagements de non-emploi de l'arme nucléaire contre les États parties à ces zones. Ces circonstances rappellent les conditions qu'elles ont formulées dans leurs déclarations unilatérales. Ainsi, en ratifiant le Protocole II du Traité de Tlatelolco, les États-Unis et le Royaume Uni avaient déclaré qu'ils reconsidéreraient leurs engagements au titre dudit protocole, en cas d'acte d'agression commis par un État partie au Traité en association avec une puissance nucléaire (29).

On retrouve cette préoccupation de défense nationale chez la France qui lors de la signature et de la ratification du Protocole II du Traité de Tlatelolco a souligné considérer qu'aucune disposition de ce protocole ou des articles du Traité auxquels il renvoie ne saurait porter atteinte au plein exercice du droit de légitime défense confirmé par l'article 51 de la Charte des Nations Unies" (30). Mais, à l'occasion de la signature des Protocoles pertinents des Traités de Rarotonga et de Pelindaba, elle reformulait sa position en déclarant que son engagement à l'égard des zones «équivaut» aux garanties négatives données par la France aux ENDAN parties au TNP (31). Cette précision est intéressante parce que elle signifie que les conditions posées dans la Déclaration française du 6 avril 1995 - critère de non-possession de l'arme nucléaire et clause de non-attaque - sont aussi valables pour les ENDAN parties à des ZEAN.

Pour sa part, l'URSS avait déclaré à l'occasion de la signature du Protocole II du Traité de Tlatelolco se réserver le droit de réexaminer sa position dans le cas ou un État partie au Traité prendrait une mesure quelconque qui soit incompatible avec ses obligations ou se livrerait à une agression. Cette formulation est tout à fait classique mais ce qui l'est moins, c'est la dernière phrase de sa déclaration dans laquelle elle précise qu'elle se réserve le droit de revenir sur sa position dans le cas ou une "action serait entreprise par un ÉDAN qui soit incompatible avec le Protocole" (32). En signant en 1986 le Protocole 2 du Traité de Rarotonga, elle a repris pratiquement la même formulation tout en précisant qu'elle entendait par action incompatible entreprise par un EDAN, l'utilisation par celui-ci "du territoire, de l'espace aérien, de la mer territoriale ou des eaux archipélagiques (des pays de la zone) pour des escales de bâtiments de la marine et

(28) Article $3, \S 1$ c) du Traité.

(29) Déclaration des États-Unis lors de la signature ( $1^{\text {er }}$ avril 1968) et de la ratification (12 mai 19/71) du Protocole additionnel II du Traité de Tlatelolco. Déclaration du Royaume-Uni lors de la signature (20 décembre 1967) et de la ratification (11 décembre 1969) du même Protocole, SIPRI Yearbook 1996, Oxford University Press, 1996, pp. 774-775.

(30) Déclaration de la France à l'occasion de la signature (18 juillet 1973) du Protocole II du Traité de Tlatelolco, Ibid.

(31) Décret $n^{\circ}$ 97-42 du 15 janvier 1997 comportant le texte des réserves et déclarations interprétatives à propos du Traité de Rarotonga et ses protocoles, Journal officiel du 22 janvier 1997, p. 1084.

(32) Déclaration de l'URSS à l'occasion de la signature (18 mai 1978) et de la ratification ( 8 janvier 1979) du Protocoles II du Traité de Tlatelolco, SIPRI Yearbook 1996, op. cit. 
d'aéronefs ayant à bord des armes nucléaires ou pour faire transiter de telles armes" (33). Cette déclaration qui n'a pas été renouvelée lors de la ratification en 1988, et que la Russie n'a pas modifié depuis est en contradiction avec l'engagements de non-emploi en premier de l'arme nucléaire que ce pays avait pris en 1982 .

En signant le Protocole II du Traité sur l'Amérique latine, la Chine s'est engagée à ne jamais employer ni menacer d'employer d'armes nucléaires contre les ENDAN parties à la zone et à ne se livrer à aucune activité incompatible avec le statut de cette zone (essais nucléaire, mise au point, installation ou déploiement terrestre, aérien ou naval d'armes nucléaires) (34). Cette position très ouverte qui contrairement à celles des autres ÉDAN ne comporte aucune condition, pourrait s'expliquer par la volonté de s'attirer la sympathie de nations du Tiers Monde auquel la Chine s'est toujours identifiée. Mais, cette souplesse chinoise est relative si on la compare à la déclaration faite par ce pays lors de la signature du Protocole 2 du Traité de Rarotonga en 1987. En effet, tout en confirmant l'engagement de non-emploi formulé antérieurement, ce pays a précisé qu'il se réservait le droit de reconsidérer ses obligations si une puissance nucléaire ou un ENDAN partie à l'accord entreprenaient une action qui constituerait une "violation flagrante" du Traité et de ses protocoles, affectant le statut de la zone et menaçant les intérêts de sécurité de la Chine (35). Ceci va à l'encontre de la position traditionnelle de ce pays en faveur du non-emploi inconditionnel d'armes nucléaires contre des ENDAN et des ZEAN réaffirmé en plusieurs occasions. Cette déclaration qui rappelle aussi celle faite par l'URSS, n'a pas été renouvelée lors de la ratification en 1989.

Ce qui est intéressant de noter dans le cas de la Chine et de la Russie c'est que l'engagement de non emploi d'armes nucléaires contre des ENDAN parties aux ZEAN peut être à tout moment remis en cause par l'une d'elles en invoquant deux raisons. D'une part cette puissance peut considérer que le comportement d'un ENDAN partie à la zone nuit aux objectifs du Traité qui l'institue. Ainsi, il suffirait qu'une seule partie se comporte d'une manière considérée comme non conforme à ses obligations pour que les assurances de non-emploi soient annulées, privant ainsi toute la zone des garanties négatives de ladite puissance. Elle peut d'autre part estimer que l'attitude d'un autre ÉDAN porterait atteinte au statut de la zone ou menacerait ses intérêts vitaux. L'ambiguïté réside ici dans le fait que la faculté d'appréciation de la situation appartient entièrement aux puissances garantes.

On voit comment en dépit de son caractère juridiquement contraignant, la renonciation à l'emploi de l'arme nucléaire contre les ZEAN peut être fragile car réversible et comportant de multiples conditions qui s'apparentent à des réserves même si par ailleurs tous les traités dont il est question ici excluent les réserves. En effet, si l'engagement de non-emploi et non menace de l'emploi clairement formulé dans les articles pertinent des protocoles implique une renonciation inconditionnelle, les déclarations faites par les ÉDAN dans le cadre des ZEAN ont pour conséquence de réduire la portée de cet engagement en posant des exceptions. On peut aussi a contrario

(33) Déclaration de l'URSS à l'occasion de la signature (15 décembre 1986) et de la ratification (98 janvier 1979) du Protocoles 2 du Traité de Rarotonga, Ibid.

(34) Déclaration de la Chine à l'occasion de la signature (21 août 1971) du Protocole II du traité de Tlatelolco, Ibid.

(35) SIPRI Yearbook 1990, p. 666. 
considérer que ces exceptions ne sauraient être assimilées à des réserves au sens de l'article 2 de la Convention de Vienne (36), car n'affectant pas fondamentalement l'effet juridique des dispositions de ces traités. Bien que la frontière entre la réserve et la déclaration interprétative soit claire in abstracto, elle le serait moins in concreto en raison d'une conception extensive de la seconde par les États dans le but de tourner les règles interdisant les réserves. Le cas d'espèce évoqué ici démontre les difficultés qu'éprouvent les puissances nucléaires à donner des garanties négatives intégrales. Pour contourner ces contraintes elles n'ont pas trouvé mieux que de déplacer le débat sur le terrain des garanties positives.

\section{Les garanties positives formulées par le Conseil de sécurité}

Dans le contexte de l'adoption du TNP, les trois États dépositaires des instruments de ratification (États-Unis, Royaume-Uni et Russie) ont proposé de fournir aux ENDAN parties au Traité des garanties positives visant à leur porter assistance s'ils étaient victimes d'une agression nucléaire. Ces garanties furent formulées dans la résolution 255 adoptée le 19 juin 1968 par le Conseil de sécurité dans le contexte de la négociation du TNP. En vertu de cette résolution, les États-Unis, le Royaume-Uni et l'URSS s'étaient engagés à fournir une assistance immédiate à tout ENDAN partie au TNP qui ferait l'objet d'une menace ou d'une attaque à l'arme nucléaire, sans toutefois que soit précisé la nature et l'ampleur de cette assistance (37).

Les insuffisances de la résolution 255 dénoncées par de nombreux PNA et surtout la perspective de difficultés risquant de surgir lors de la Conférence sur l'avenir du TNP prévue la même année pourraient expliquer l'adoption le 11 avril 1995, par le Conseil de sécurité, sur la base d'un projet des cinq membres permanents, d'une nouvelle résolution sur des garanties positives. Cette initiative constituait une manœuvre de diversion particulièrement habile des ÉDAN qui sans céder sur le fond des garanties négatives faisait miroiter aux ENDAN un renforcement des garanties positives, beaucoup moins coûteuses politiquement. La résolution 984 (1995) stipule qu'«en cas d'agression ou de menace d'agression avec emploi d'armes nucléaires" contre un ENDAN partie au TNP, le Conseil de sécurité devra "prendre des mesures urgentes afin de fournir, conformément à la Charte, une assistance à l'État victime». Le texte invite les États membres «à prendre, individuellement ou collectivement, les mesures appropriées en vue de répondre à une demande de la victime en matière d'assistance technique, médicale, scientifique ou humanitaire". Il évoque enfin l'adoption de "procédures appropriées" pour répondre à "toute demande" de la victime et en particulier «le versement d'une indemnité par l'agresseur, conformément au droit international, en réparation des pertes, dommages ou préjudices subis du fait de l'agression " (38).

(36) L'article $2 \S 1 \mathrm{~d}$ ), de la Convention définit la réserve comme une déclaration unilatérale par laquelle un État "vise à exclure ou à modifier l'effet juridique de certaines dispositions du traité dans leur application à cet État".

(37) La résolution 255 fut adoptée par 10 voix contre 0 et 5 abstentions (Algérie, Brésil, France, Inde et Pakistan). Dans ce texte le Conseil "reconnaît qu'une agression avec emploi d'armes nucléaires ou la menace d'une telle agression à l'encontre d'un État non doté d'armes nucléaires créerait une situation dans laquelle le Conseil de sécurité et, au premier chef, tous ses membres permanents dotés d'armes nucléaires devraient agir conformément à leurs obligations aux termes de la Charte des Nations Unies"

(38) Résolution 984 (1995), en particulier $\$ 3$ à 6. 
En proposant la résolution 984 , les membres permanents visaient deux objectifs : réactualiser la résolution 255 (1968) et ce faisant, préciser les garanties positives en faveur des ENDAN parties au TNP. Il faut remarquer ici que curieusement, la résolution 984 ne fait nulle part mention de la résolution 255 dont elle s'inspire pourtant. Cet oubli volontaire trouverait son explication dans le peu d'enthousiasme qu'a soulevé le texte de 1968 chez les États concernés. Une autre explication est que la résolution 255 étant antérieure à l'adoption du TNP, elle n'y faisait pas mention. Or les puissances nucléaires sont très attachées à ce que les garanties de sécurité, qu'elles soient positives ou négatives, ne soient octroyées qu'aux ENDAN parties au TNP. La résolution 984 a comblé cette lacune, mais elle marque surtout un progrès sur trois plan : elle a été adoptée par les cinq membres permanents du Conseil de sécurité (39); elle traite de la question de l'assistance technique et humanitaire à l'État victime de manière plus précise; elle évoque enfin le versement d'une indemnité par l'agresseur en réparation des dommages causés.

Les aspects positifs ont été soulignés par les ÉDAN. Ainsi, les États-Unis ont considéré que les garanties positives figurant dans cette résolution sont "réalistes, sérieuses et applicables", elles sont de leur point de vue la «seule base sur laquelle peuvent se fonder des garanties de sécurité crédibles" (40). La France et le Royaume-Uni ont pour leur part insisté sur le caractère novateur du texte qui pour la première fois apporte une "réponse globale, collective et concrète" aux aspirations des ENDAN (41). Les trois puissances occidentales ont affirmé par ailleurs qu'elles ne pouvaient pour des raisons liées à leurs conceptions de sécurité aller au-delà de l'engagement contenu dans la résolution 984 . On peut comprendre qu'elles soient dans ce domaine constamment préoccupées par le fait que des garanties positives précises et contraignantes auraient pour conséquence de les impliquer dans un scénario de conflit nucléaire qu'elles n'auraient pas déclenché. Des garanties positives renforcées auraient ainsi les mêmes implications qu'une dissuasion élargie, concept à l'égard duquel les puissances nucléaires restent très réservées.

En dépit des innovations qu'elle introduisait en matière de garanties positives, la résolution 984 à l'instar de celle qui l'avait précédée n'est pas exempte de critiques. On peut mentionner parmi celles-ci, le fait qu'elle n'apporte rien de nouveau par rapport aux dispositions du Chapitre VII de la Charte en cas d'agression. Les garanties positives seraient en quelque sorte superflues parceque elles ne créent, par rapport à la Charte, aucune nouvelle obligation. En outre, l'hypothèse retenue dans la résolution 984 est que l'agression nucléaire dont il est fait mention n'est pas censée provenir des membres permanents du Conseil de sécurité.

La critique la plus sévère a émané de l'Inde qui a rappelé le caractère discriminatoire de la résolution 984 qui ne s'appliquant qu'aux ENDAN parties au TNP, signifierait l'autorisation implicite donnée aux ÉDAN d'utiliser l'arme nucléaire contre les ENDAN non parties (42). Mais, à l'exception

(39) La France s'était abstenue lors du vote de la résolution 255 estimant que «les nations du monde ne recevront les garanties de sécurité auxquelles elles sont en droit de prétendre que lorsque les puissances nucléaires auront accepté de s'engager dans la voie du désarmement nucléaire et auront mené celui-ci à son terme" (document S/PV.1430, § 51). La Chine populaire n'était pas encore à l'époque membre des Nations Unies, son siège étant occupé par Taïwan.

(40) S/PV.3514, p. 28, (11 avril 1995).

(41) Ibid., pp. 29-30.

(42) Ibid., p. 42. 
de l'Inde, la plupart des critiques adressées à la résolution 984 portaient sur les dispositions proprement dites du texte. On a notamment souligné que les garanties positives prévues dans la résolution seraient inapplicables en raison du droit de veto des cinq puissances nucléaires du Conseil de sécurité. A ce propos, l'Égypte avait suggéré, sans succès, que la mise en œuvre de ces garanties soit explicitement "à l'abri du champ d'application du droit de veto afin d'en assurer la crédibilité " (43). Mais on pourrait penser que la procédure prévue à l'article 3 du dispositif de la résolution en cas d'agression ou menace d'agression par l'arme nucléaire équivaut à mettre entre parenthèse le droit de veto. En effet la référence "aux mesures urgentes" ainsi qu'au devoir des membres permanents de "porter immédiatement la question à l'attention du Conseil» et de "s'employer à obtenir» de celui-ci la fourniture de "l'assistance nécessaire à l'État victime", exprime l'impératif d'une action du Conseil.

On pourrait aussi objecter que l'emploi et la menace d'emploi de l'arme nucléaire, constitue une menace grave à la paix mondiale et par ce fait devrait relever automatiquement de la procédure du Chapitre VII et non d'une para-procédure instituée par une résolution du Conseil de sécurité. On peut s'étonner que cette résolution qui vise à porter assistance aux États victimes d'une agression nucléaire ne mentionne pas dans son dispositif qu'une agression à l'arme nucléaire contre un ENDAN partie au TNP constitue une menace contre la paix et la sécurité internationales qui déclencherait l'adoption immédiate de mesures par le Conseil, conformément aux articles 39 à 49 du Chapitre VII (44).

Se pose donc la question des sanctions à mettre en cuvre contre une telle agression. A ce propos, on peut imaginer l'application de sanctions à l'égard d'un État procédant à une attaque nucléaire contre ses voisins (45). Le but recherché ici est de dissuader l'utilisation de l'arme nucléaire, en adressant un message fort à destination d'un État tenté d'utiliser cette arme. Cette mesure s'inscrit évidemment dans l'hypothèse d'un conflit ou un État autre que les cinq membres permanents du Conseil aurait l'intention d'employer des moyens nucléaires contre un ENDAN. Il s'agit donc d'un contexte de conflit régional susceptible de se nucléariser, ce qui peut potentiellement être le cas au Moyen-Orient et en Asie du Sud. Mais peut-on envisager que les sanctions militaires prévus aux articles 41 à 48 de la Charte soient de nature nucléaire? Sur le plan juridique rien dans le Chapitre VII de la Charte ne pourrait être interprété comme interdisant des sanctions militaires avec usage d'armes nucléaires, d'une part parce que la Charte ne fait aucunement la distinction entre les différentes catégories d'armes à employer dans le contexte de ces sanctions, et d'autre part du fait que l'arme nucléaire est postérieure à ce document. Si une telle hypothèse peut être envisagée, le Conseil de sécurité serait l'organe habilité à décider et à faire appliquer des sanctions collectives, conformément à la mission centrale de maintien de la paix et de la sécurité internationale que lui confie l'article 24 de la Charte.

(43) Ibid., p. 9 à 13 .

(44) La résolution 984 mentionne simplement dans son préambule et en termes conditionnels "qu'au sens où l'entendent les dispositions pertinentes de la Charte des Nations Unies, toute agression avec emploi d'armes nucléaires mettrait en danger la paix et la sécurité internationales ".

(45) Cette proposition a été faite dans une Étude d'experts des Nations Unies intitulée Effective and Verifiable Measures Which Would Facilitate the Establishment of a Nuclear-weaponfree Zone in the Middle East, Rapport du Secrétaire général des Nations Unies, document, A/45/435, $\$ 139$. 
Sans entrer dans la discussion sur les modalités de l'exécution des sanctions nucléaires, il est utile de mentionner le récent débat d'experts sur les armes nucléaires au service de la sécurité collective; certains n'hésitant pas à envisager la mise en place d'une force nucléaire internationale sous le contrôle du Conseil de sécurité des Nations Unies (46). Mais quel serait l'intérêt de sanctions nucléaires si l'agression a déjà eu lieu ? Il parait évident que l'arme nucléaire dans le cadre de la sécurité collective viserait moins à répondre à une agression nucléaire qu'à la prévenir. A l'instar de la dissuasion, elle relèverait moins d'une logique d'emploi que d'une logique de menace de l'emploi. Cependant, l'hypothèse de sanctions nucléaires collectives soulève des questions juridiques et politiques extrêmement complexes qui concernent notamment l'accord préalable des puissances nucléaires, la redéfinition des doctrines de dissuasion, le statut d'une force nucléaire internationale. Outre l'ampleur des difficultés à résoudre, il s'agira d'une véritables révolution psychologique dans les perceptions actuelles très manichéennes de l'arme atomique. En définitive ce scénario n'est pas souhaitable car il contribuera à donner une légitimité nouvelle aux armes nucléaires et de ce fait irait à contre-courant d'une tendance générale en faveur de l'objectif du désarmement nucleaire.

On voit que la question des garanties positives soulève des préoccupations et des problèmes complexes de sécurité auxquels la résolution 984 a partiellement répondu. Tout en relevant des aspects positifs dans le texte, la majorité des PNA a estimé que ladite résolution constitue "une démarche initiale sur la voie d'une élaboration multilatérale" portant sur un "engagement ferme à ne pas recourir aux armes nucléaires" (47). On pourrait interpréter cette position comme signifiant que la véritable solution au besoin de sécurité des ENDAN réside moins dans l'assistance à la victime d'une attaque nucléaire que dans l'interdiction de l'emploi même de l'arme nucléaire contre ces États. Ils posent ainsi la primauté des garanties négatives sur les garanties positives comme l'illustre par ailleurs leur activisme dans la recherche d'une formule commune à cet effet.

\section{II. - LES DIFFICULTÉS DE L'ÉLABORATION D'UNE FORMULE COMMUNE}

Des discussions sont engagées depuis 1979 dans le cadre d'un comité spécial de la Conférence du désarmement de Genève en vue de définir une formule commune visant à interdire l'emploi ou la menace de l'emploi d'armes nucléaires contre des ENDAN. Le terme formule commune utilisé dans les résolutions de l'Assemblée générale et les rapports de la Conférence du désarmement n'a pas précisément été défini. La formule commune pourrait signifier la formulation de garanties de sécurité conjointement ou collectivement par toutes les puissances nucléaires. L'élaboration de cette formule

(46) Voir en particulier Michael MAZARR \& Alexander Lennon, Toward A Nuclear Peace, The Future of Nuclear Weapons, St Martin's Press (New York), 1994, Chapitre 7, pp. 96-98. Voir aussi Jean-François DaguZan, "L'arme nucléaire française et la question des garanties nucléaires dans un système de sécurité collective", in : Demain l'ombre portée : la dissuasion nucléaire en question, CREST, (Paris) : École Polytechnique, 1997, pp. 116-120.

(47) Déclaration de l'Indonésie au nom du Mouvement des Non-alignés, Documents d'actualité internationale, $\mathrm{n}^{\circ} 12,15$ juin $1995, \S 171$. 
commune est d'autant plus difficile qu'elle implique un accord entre deux approches contradictoires quant à la méthode et à l'objectif recherché dans la définition des garanties négatives. L'une prône que la formule commune devra consacrer des garanties inconditionnelles et d'application universelle et qu'elle sera insérée dans un instrument international ayant une force juridique obligatoire. L'autre au contraire privilégie un engagement unilatéral de non-emploi contre les ENDAN qui réponde à la diversité des situations ct des bcsoins en matière de sécurité des différentes catégories d'États et qui ne nécessite pas un formalisme juridique trop rigide. La première approche est défendue par les PNA tandis que les puissance nucléaires (à l'exception de la Chine) se font les avocats de la seconde.

S'il n y a pas d'objection de principe à l'idée d'une formule commune, de sérieuses difficultés existent concernant la portée et la forme de l'arrangement contenant cette formule ainsi que sur les critères permettant de définir les bénéficiaires des garanties négatives.

\section{A. Portée de l'arrangement contenant la formule commune}

La Comité spécial de la Conférence du désarmement avait considéré qu'un accord sur la portée des arrangements pourrait faciliter un accord sur la forme de ceux-ci. A ce propos, trois sortes d'engagements ont été proposés jusqu'ici qui concernant respectivement l'interdiction du recours à l'arme nucléaire, le non-emploi en premier et le non-emploi inconditionnel de ces armes contre les ENDAN. Contrairement à la dernière, les deux premières questions sont examinées par la Conférence du désarmement au titre d'un point de l'ordre du jour distinct intitulé "prévention de la guerre nucléaire, y compris toutes les questions qui y sont liées". Elles sont mentionnées ici parce qu'elles ont une incidence sur les garanties négatives de sécurité.

En 1961, l'Assemblée générale des Nations Unies adoptait une résolution dans laquelle elle considérait l'emploi de l'arme nucléaire comme "un crime contre l'humanité et la civilisation"(48). Elle demandait au Secrétaire général de procéder à une consultation des États sur la possibilité de convoquer une conférence internationale chargée d'adopter une convention sur l'interdiction de l'arme nucléaire. S'inspirant de cette résolution aux termes sans équivoque, l'Inde avait déposé en 1982 à l'Assemblée générale un projet de Convention sur l'interdiction de l'utilisation des armes nucléaires. En vertu de l'article premier de ce texte les ÉDAN «s'engageraient solennellement à ne pas utiliser, ni menacer d'utiliser les armes nucléaires en aucune circonstance» (49). C'est donc une interdiction générale et inconditionnelle qui est proposée ici. L'argument somme toute assez convaincant développé par l'Inde en direction des ENDAN est que la meilleure garantie de non-emploi de l'arme nucléaire contre eux réside dans un régime de prohibition totale du recours et non dans des déclarations unilatérales qui offriraient une «illusion de sécurité»(50).

(48) Résolution 1653 (XVI) du 24 novembre 1961, voir aussi les résolutions 33/71 B (14 décembre 1978), 34/83 G (11 décembre 1979), 35/152 D (12 décembre 1980), 36/92 I du 9 décembre $1981,45 / 59$ B du 4 décembre 1990.

(49) Assemblée générale, deuxième Session spéciale sur le désarmement (1982), A/S12/AC.1/13 et A/S-12/AC.1/L.4.

(50) CD/1281, § 14. Voir aussi Jasjit SinGH, "La non-prolifération nucléaire au xxe siècle, Politique étrangère, 3/95, automne 1995, pp. 638 . 
L'Inde est ainsi l'inspirateur de la résolution adoptée chaque année par l'Assemblée générale qui comporte en annexe le projet de convention (51). À l'occasion du vote de cette résolution, la Chine est la seule puissance nucléaire à appuyer la revendication, la Russie s'abstient tandis que les ÉtatsUnis, la France et le Royaume-Uni se trouvent dans la liste des opposants au côté de leurs alliés de l'OTAN, Les trois puissances nucléaires occidentales rejettent en effet cette proposition considérant que le recours à la force contre l'intégrité territoriale des États ou leur indépendance politique - quelque soit le type d'armement utilisé - était déjà prohibé dans l'article $2 \$ 4$ de la Charte des Nations Unies et que par conséquent il n'y avait nul besoin d'une interdiction du recours à la force concernant l'arme nucléaire. Il y a ici manifestement une volonté des puissances occidentales de cantonner les discussions sur le non-emploi de l'arme nucléaire dans le cadre de la prévention de la guerre en général (52).

Mais une interdiction éventuelle de l'emploi de l'arme nucléaire ne constituerait pas en soi un précédent, un régime de non-emploi avait été institué dans le Protocole de Genève de 1925 pour les armes bactériologiques et chimiques (53). Cette interdiction démontre comment la logique du droit des conflits armés et du droit humanitaire (jus in bello) peut prévaloir sur la logique du recours à la force (jus ad bellum). Les partisans de l'illicéité du recours à l'arme nucléaire font valoir que l'emploi de cette arme en raison de ses effets indiscriminés sur les combattants et les populations civiles était par conséquent contraire aux règles du droit des conflits armés et du droit humanitaire contenues dans la Convention IV de La Haye et son Règlement (1907) ainsi que dans les quatre Conventions de Genève de 1949 et leurs deux Protocoles additionnels de 1977 (54). D'autres estiment que l'emploi de l'arme nucléaire pourrait faire l'objet de limitations au nom des principes du droit des conflits armés, tout en permettant le jeu de la dissuasion, nécessaire à la sécurité (55). Ces préoccupations transparaissent dans l'avis consultatif du 8 juillet 1996. Si la Cour a estimé que «ni le droit international coutumier, ni le droit international conventionnel ne comportent d'interdiction complète et universelle de la menace ou de l'emploi des armes nucléaires en tant que telles", elle a toutefois considéré que "la menace ou l'emploi d'armes nucléaires serait généralement contraire aux règles du droit international applicable dans les conflits armés, et spécialement aux principes et règles du droit humanitaire" (56).

(51) Projet de résolution de l'Inde (A/C.1/52/L.15) à l'origine de la résolution $52 / 39 \mathrm{C}$ adoptée le 9 décembre 1997 par 109 voix contre 30 et 27 abstentions.

(52) Voir le projet de résolution sur la "prévention de la guerre nucléaire et ensemble des questions connexes, prévention de la guerre à l'ère nucléaire" présenté par l'Allemagne et appuyé par 8 pays membres de l'OTAN, 3ye session de l'Assemblée générale, A/C.1/39/L.40 et Kev.1.

(53) La Convention de 1993 sur les armes chimiques interdit ausi bien l'emploi que la mise au point, la fabrication et le stockage.

(54) Voir notamment Jozef GoldBlatT, «Rendre illégales les armes nucléaires », in Un monde sans armes nucléaires, Conférence Pugwash sur la science et les affaires mondiales, L'Age d'homme (Paris), 1995, pp. 205-219. Pour une critique de la thèse de l'illicéité de l'emploi voir notamment Jean-François Guilhaudis, "Armes nucléaires et sécurité collective, remarques sur la réglementation des armes nucléaires ", in : J.-F. Gull.haudis et M. Torelli (éd.), Forces armées et diplomatie, vol. II, Martinus Nijhoff Publishers (Dordrecht-Boston-Lancaster), 1985, pp. 151-157.

(55) Voir à ce propos la thèse des deux ordres juridiques qui s'appliqueraient à l'arme nucléaire, celui de la possession et celui de l'emploi, in : Henry Meyrowitz, "Problèmes juridiques relatifs à l'arme à neutron", $A F D I, 1981$, pp. 87-125 (en particulier pp. 93-94).

(56) Licéité de la menace ou de l'emploi de l'arme nucléaire, CIJ, Rec. 1996, p. 51, § 105. 
Face à l'opposition résolue manifestée par les puissances nucléaires occidentales sur la question, une alternative fut proposée, le non-emploi en premier. Dès 1966, année ou elle avait procédé à son premier essai nucléaire, la Chine avait déclaré qu'elle "ne serait jamais, et en aucune circonstance, la première à utiliser des armes nucléaires, un engagement qu'elle a renouvelé depuis en appelant à la conclusion d'une convention internationale à cet effet (57). L'exemple chinois sera suivi en 1982 par l'URSS qui s'est solennellement engagée devant l'Assemblée générale réunie en session extraordinaire à ne pas utiliser en premier l'arme nucléaire (58).

L'argument invoqué souvent est qu'un engagement de non-emploi en premier améliorerait la sécurité de tous les pays, et servirait mieux la cause de la non-prolifération que des assurances sélectives données en récompense à la participation au TNP. Les partisans du non-emploi en premier font aussi valoir qu'un tel engagement équivaudrait de facto à une garantie négative de sécurité pour les ENDAN, qui étant dépourvus d'armes nucléaires, ne peuvent s'exposer à des représailles. A ce propos, le Comité spécial de la Conférence du désarmement a examiné "la pertinence et les incidences directes" du non-emploi en premier pour ce qui est des garanties négatives de sécurité (59). A l'occasion des discussions, les États-Unis, la France et le Royaume-Uni ont contesté l'idée qu'il y ait un rapport entre la question du non-emploi en premier et celle des garanties de sécurité (60). On pourrait en effet considérer que l'engagement de non-emploi en premier ne peut constituer une garantie de sécurité effective et crédible pour les ENDAN parce que sa validité erga omnes pouvait être remise en cause, à tout moment, par les agissements d'une autre puissance nucléaire. Le scénario au cours duquel un ÉDAN procéderait à un tir nucléaire à partir du territoire terrestre ou maritime d'un ENDAN, exposant ainsi ce dernier à une riposte nucléaire aussi peu probable soit-il n'en est pas moins possible. L'argument généralement invoqué par les opposants à l'interdiction de l'emploi comme au nonemploi en premier pose que rien dans la Charte ne limiterait le droit des États d'employer les moyens qu'ils jugent les plus appropriés dans l'exercice de leur droit de légitime défense. La réaction négative des puissances occidentales s'expliquerait par la crainte qu'un engagement de non-emploi en premier n'affaiblisse la dissuasion qui suppose au contraire que demeure libre de contraintes la voie de l'emploi. Ces puissances voudraient garder la possibilité d'un recours à l'arme suprême quelque soit le moyen d'agression utilisé contre elles (61).

Le non-emploi en premier permet l'emploi en second ou en riposte à une agression nucléaire. Si le non-emploi en premier était accepté par toutes les puissances concernées, cela signifierait que le rôle de leurs armes nucléaires est uniquement de prévenir une attaque nucléaire. La dissuasion n'est donc pas remise en cause fondamentalement, seulement elle ne jouerait plus

(57) $\mathrm{CD} / 1309 \mathrm{du} 5$ avril 1995. Voir aussi sur la position chinoise sur le non-emploi en premier : Dingli SHEN, "Toward a nuclear-weapon-free world : a Chinese perspective", The Bulletin of the Atomic Scientists, vol. 50, n ${ }^{\circ} 2$, mars-avril 1994, pp. 51-54.

(58) A/S-12/PV.12. Voir à ce propos Jean-François Guilhaudis, "Les puissances nucléaires et la deuxième session extraordinaire de l'AGNU sur le désarmement", Arès Défense et sécurité, Supplément $\mathrm{n}^{\circ} 1,1983, \mathrm{pp} .56-84$.

(59) $\mathrm{CD} / 825$, p. 212 .

(60) CD/540, p. 172 . Voir aussi CD/825, p. 219.

(61) Voir les thèses opposées de Henry Meyrowitz, «Le débat sur le non-recours en premier aux armes nucléaires, op. cit. et Jean-François GuilHaudis, "Armes nucléaires et sécurité collective ", op. cit. 
contre une attaque par des moyens conventionnels, mais s'exercerait pleinement à l'égard d'une velléité d'agression nucléaire. Ce type de mesure consoliderait certainement le régime de non-prolifération parce qu'il limiterait chez les ENDAN l'attrait pour l'arme suprême dont l'utilité serait circonscrite à la prévention d'une guerre nucléaire entre les grandes puissances (62).

L'échec des propositions visant l'interdiction du recours à l'arme nucléaire et le non-emploi en premier a conforté la position de ceux des ENDAN qui restent convaincus qu'une réponse réaliste et crédible à leurs besoins de sécurité réside dans des garanties négatives exemptes de toutes conditions. En effet, l'analyse des travaux de la Conférence du désarmement révèle que la majorité des ENDAN membres sont favorables à l'adoption d'un «instrument à caractère contraignant portant sur des garanties de sécurité efficaces, inconditionnelles et complètes"(63). Pour les PNA «la formule commune retenue dans la convention devrait être claire, crédible et dénuée d'ambiguïté, et devrait répondre aux préoccupations de toutes les parties" (64). Ils insistent pour que les ÉDAN définissent dans la formule commune des garanties négatives de sécurité harmonisées et inconditionnelles. Harmonisées mais elles s'inspireraient de celles données par la Chine. Inconditionnelles parceque elles ne comporteraient aucune réserve, ne seraient pas sujettes à des interprétations divergentes et devraient avoir une portée, une application et une durée illimitées.

Face à cette revendication, les puissances nucléaires occidentales invoquent encore l'argument de la dissuasion pour refuser d'aller au-delà des engagements unilatéraux qu'elles ont déjà formulés. Elles soulignent que des garanties négatives de sécurité inconditionnelles affaibliraient la capacité dissuasive de leurs armes nucléaires. Ceci aurait pour conséquence selon elles d'accroître le risque d'une agression par des moyens conventionnels menée par un ENDAN avec l'appui d'une puissance nucléaire (65). L'ampleur des divergences sur le caractère inconditionnel ou non des garanties négatives explique que le Comité spécial de la Conférence du désarmement n'ait pu véritablement mener des négociations de fond sur la portée de la formule commune. Le même constat pourrait être fait concernant la forme de l'engagement portant formule commune de non-emploi.

\section{B. Forme de l'arrangement contenant la formule commune}

Si elle s'avère moins insurmontable que la question de la portée des arrangements, celle sur leur nature se heurte néanmoins à des difficultés.

(62) Différents scénarios possibles relatifs à l'adoption du non-emploi en premier par les États-Unis sont envisagés dans David Gompert, Kenneth WatMan \& Dean Wilkening, US Nuclear Declaratory Policy, The Question of Nuclear First Use, Rand (Santa Monica, California), 1995, pp. 1-24.

(63) Communiqué final de la réunion du Bureau de coordination des PNA (Bandoeng, 27 avril 1995), Annexe.

(64) Déclaration du Groupe des 21 sur la question des garanties négatives de sécurité; $\mathrm{CD} / 1256$, p. 2 (31 mars 1994).

(65) Voir à ce propos les arguments développés par un groupe d'experts américains in: Nuclear Proliferation, Confronting the New Challenges, Report of an Independant Task Force on Nuclear Proliferation? Council of Foreign Relations (New York), 1995, p. 23. 
Les membres de la Conférence du désarmement restent divisés sur la forme de l'arrangement international sur les garanties négatives. Quatre solutions ont été envisagées : un protocole additionnel au TNP, une résolution du Conseil de sécurité, des accords portant garanties de sécurité individualisées, une convention internationale.

En dépit des souhaits exprimés par le groupe des PNA au sein du Comité des dix-huit puissances sur le désarmement, le TNP fut adopté le $1^{\mathrm{er}}$ juillet 1968 sans aucune disposition relative à des garanties de sécurité pour les ENDAN (66). C'est au cours de la Conférence des États non-dotés de l'arme nucléaire réunie en août 1968 en vue d'examiner les moyens de garantir ces États contre l'emploi ou la menace de l'emploi d'armes nucléaires, que furent examinées les premières propositions visant notamment la conclusion d'un protocole additionnel au TNP portant sur les garanties de sécurité (67). L'appel en faveur d'un protocole additionnel au TNP fut renouvelé au cours des première (1975) et quatrième (1990) Conférences d'examen du TNP sans plus de succès (68).

La méthode du protocole présente l'avantage d'éviter la procédure complexe et longue de l'amendement du dispositif du Traité lui-même qui avait été un moment envisagé. En outre, l'adoption éventuelle d'un protocole au TNP sur des garanties négatives ne constitue pas en soi une innovation. Le Traité de Tlatelolco (1967) portant renonciation à l'acquisition d'armes nucléaires par les pays d'Amérique latine a été complété par un Protocole additionnel contenant un engagement des puissances nucléaires de non-emploi d'armes nucléaires contre les États parties à ce Traité. Le modèle existe donc déjà, on pourrait imaginer qu'un protocole au TNP soit rédigé dans les mêmes termes. Comme dans le cadre des ZEAN, les ÉDAN auraient la possibilité dans des déclarations accompagnant la signature et la ratification de préciser les conditions dans lesquelles elles entendent accorder leurs garanties négatives.

Si la formule du protocole présente l'intérêt de rééquilibrer les droits et obligations des parties au TNP, les bénéfices escomptés semblent hasardeux. En effet, cette opération comporte aussi le risque d'ouvrir une boite de Pandore, de mettre à plat tout le Traité au moment ou celui-ci venait d'être prorogé pour une période indéfinie. Le TNP étant le fruit d'un consensus fragile, modifier aujourd'hui son équilibre risque d'en compromettre l'avenir. Un protocole additionnel au TNP n'est en rien acquis comme l'ont révélé les concertations menées jusqu'ici. Cette question fut examinée à la Conférence du désarmement à l'initiative du Nigeria qui a soumis un projet d'accord sur l'interdiction de l'emploi ou de la menace d'emploi d'armes nucléaires

(66) Voir le Mémorandum commun du groupe des PNA, ENDC 178. Si l'URSS s'est déclarée favorable à inclure dans l'instrument une disposition interdisant l'emploi d'armes nucléaires contre des ENDAN parties au Traité et qui ne disposeraient pas d'armes nucléaires sur leur territoire, les États-Unis et le Royaume-Uni s'y sont opposés (in Les Nations Unies et le désarmement 1970-1975, Publication des Nations Unies (New York), 1976, pp. 283 à 296).

(67) Voir à ce propos les projets de résolutions de pays d'Amérique latine (A/Conf.35/C.1/L.3/Rev.1 et 2), d'Afrique (A/Conf.35/C.1/L.4) et du Pakistan (A/Conf.35/C.1/L.11). La résolution $\mathrm{A}$ de la Conférence $(\mathrm{A} / 7277)$ qui s'inspirait dans sa formulation d'un projet de la République fédérale allemande (A/Conf.35/C.1/L.13/Rev.1 se caractérise par une extrême modération, se limitant à réaffirmer le principe du non-emploi de la force avec ou sans armes nucléaires conformément à l'article $2 \S 4$ de la Charte des Nations Unies et demandait aux ÉDAN de s'engager à mettre en œuvre ce principe.

(68) Voir les projets de résolution de la Roumanie (NPT/CONF.1/22) et du Nigeria (NPT/CONF.IV/17). 
contre les ENDAN parties au TNP (69). Ce projet. et d'une manière générale les propositions de protocole au TNP n'ont semble-t-il pas obtenu un succès notable au cours des discussions (70). Trois raisons pourraient expliquer cet échec. Premièrement, les PNA eux-mêmes sont divisés sur l'opportunité d'un protocole additionnel au TNP, ainsi l'Inde récuse l'idée d'un lien entre le TNP et les garanties de sécurité, estimant que ces dernières "ne sauraient, ni ne devraient être considérées comme contrepartie de la signature du $T N P »(71)$. Deuxièmement, la majorité des ENDAN continuent d'accorder la priorité à une convention internationale spécifique comportant un engagement inconditionnel et d'application universelle. Troisièmement, il y a bien sûr l'opposition traditionnelle de certaines puissances nucléaires qui veulent conserver le TNP en l'état, considérant que les États parties à ce Traité bénéficient déjà des engagement de non-emploi formulés dans les déclarations unilatérales. La Conférence de 1995 qui a décidé en vertu de la procédure prévue à l'article $10 \S 2$ de proroger pour une durée indéterminée le TNP a définitivement clos ce dossier. En effet, tout en reconnaissant l'importance de la question des garanties de sécurité au profit des États parties, elle ne mentionne nulle part que la réponse à cette question soit apportée dans le cadre du Traité lui-même (72).

On a également proposé que l'arrangement portant formule commune sur les garanties négatives pourrait prendre la forme d'une résolution du Conseil de sécurité. Ainsi, les Pays-Bas ont soumis en 1981 dans un document de travail présenté à la Conférence du désarmement, les termes d'une formule commune sur les garanties négatives à inclure dans une résolution du Conseil de sécurité. La formulation retenue dans le projet s'inspire des déclarations unilatérales américaines et britanniques (73). Cette approche a été une nouvelle fois envisagée en 1994 par le Groupe occidental à la Conférence du désarmement (74). Mais, la disparité actuelle des positions des ÉDAN sur les garanties négatives, du fait de la position en flèche de la Chine, rend cet exercice particulièrement ardu.

La résolution du Conseil de sécurité en tant qu'acte unilatéral d'une organisation internationale qui s'imposerait à tous les États membres conformément à l'article 25 de la Charte et à une opinion de la Cour (75), présente toutefois un caractère juridiquement moins solennel et impératif qu'une convention internationale. En cela elle pourrait paraître moins attractive pour de nombreux ENDAN. Mais, l'élément le plus significatif ici est qu'une résolution du Conseil de sécurité implique le transfert vers cet organe et donc vers les membres permanents des discussions sur les garanties néga-

(69) $\mathrm{CD} / 967$.

(70) Le Rapport de la Conférence du désarmement sur sa session de 1994, ne fait que mentionner la proposition de protocole sans autres commentaires, CD/1281, p. 142, § 13 .

(71) S/PV.3514, p. 42.

(72) NPT/CONF.1995/L.5 \$8.

(73) Dans le projet de résolution proposé, le Conseil de sécurité aurait pris acte de l'engagement solennel des EDAN de ne pas utiliser ou menacer d'utiliser des armes nucléaires contre un ENDAN qui se serait engagé à ne pas mettre au point ou acquérir des armes nucléaires, étant entendu que cet État ne se livre pas à une attaque contre un ÉDAN ou ses alliés avec l'appui d'un autre ÉDAN (CD/SA/WP.6 et 9).

(74) $\mathrm{CD} / 1281$, p. $144, \S 21$.

(75) L'article 24 stipule que "les membres de l'Organisation conviennent d'accepter et d'appliquer les décisions du Conseil de sécurité conformément à la présente Charte". S'appuyant sur une interprétation extensive de cet article, la CIJ a reconnu dans l'avis consultatif du 21 juin 1971 relatif à la Namibie, le caractère obligatoire des résolutions du Conseil de sécurité, Recueil CIJ, 1971 , pp. 52-54. 
tives actuellement menées au sein de la Conférence du désarmement. Beaucoup d'ENDAN n'y sont pas disposés. Ainsi, les PNA tout en exprimant des doutes quant à l'importance d'assurances de sécurité formulées dans une résolution du Conseil de sécurité, aussi bien intentionnées soit elle, insistent pour que la formule commune résulte d'une négociation dans le cadre du Comité spécial de l'organe multilatéral de Genève (76). Pour le Pakistan, la Conférence du désarmement «est la seule instance appropriée pour l'examen des garanties de sécurité"(77). On peut comprendre que pour ces pays, l'examen des garanties négatives au sein de l'organe multilatéral de Genève constitue l'assurance que leurs intérêts y seront mieux préservés qu'au Conseil de sécurité. Certains ENDAN sont cependant disposées à envisager une telle solution mais à titre intérimaire seulement en attendant l'adoption d'un instrument international.

En dehors d'une résolution du Conseil de sécurité, les ÉDAN semblent donner leur préférence - s'il y avait une hiérarchie d'options - à des engagements de non-emploi au cas par cas. Dans cette approche on prône l'élaboration de formules spécifiques ( $\grave{a}$ la carte») répondant à la diversité des situations et des besoins en matière de sécurité parmi les ENDAN. C'est ainsi que fut envisagée l'opportunité de formuler des garanties négatives individualisées. Ces garanties individualisées pourraient être accordées soit dans un cadre bilatéral (un ÉDAN + un ENDAN) soit dans un cadre plurilatéral (les ÉDAN + un ENDAN).

L'URSS s'était déclarée disposée en 1982 «à conclure des accords de garanties bilatéraux avec les États qui ne possèdent pas d'armes nucléaires et n'en ont pas sur leur territoire" (78). Cette proposition qui doit être située dans le contexte de la Guerre froide n'avait reçu pour des raisons différentes ni l'appui des États occidentaux, ni celui des PNA. Les premiers ont invoqué son caractère propagandiste, les seconds pour des raisons liées à leur méfiance traditionnelle à l'égard du bilatéralisme qu'ils considèrent politiquement moins équitable que le multilatéralisme. La bilatéralisation des garanties de sécurité présente l'avantage d'être plus facilement négociable, mais elle comporte aussi l'inconvénient de multiplier les accords de garanties, posant ainsi le problème de leur harmonisation. L'approche bilatérale a été proposée plus récemment dans le cadre de l'objectif de la dénucléarisation de la péninsule coréenne. Ainsi, dans l'accord cadre signé le 21 octobre 1994 entre les États-Unis et la Corée du Nord, les premiers déclarent qu'ils fourniront à la seconde «des assurances formelles contre la menace ou l'usage d'armes nucléaires par les États-Unis"(79). On est ici en présence d'une déclaration d'intention des États-Unis et à ce jour cette intention ne s'est pas encore concrétisée, les conditions posées dans l'accord bilatéral de 1994 n'ayant pas été remplies.

Des garanties négatives individualisées mais formulées par l'ensemble des ÉDAN dans un document commun sont également possibles. Cette approche a été adoptée dans le cadre des mémorandums sur les garanties de sécurité en liaison avec l'adhésion au TNP de la Bélarus, du Kazakhstan et de l'Ukraine. Ces mémorandums signés le 5 décembre 1994 entre les États-Unis, le Royaume-Uni et la Russie d'une part et chacune des trois

(76) $\mathrm{CD} / 1256$, p. 2.

(77) S/PV.3514, p. 14.

(78) A/S-12/P.V. 12 , pp. $22-25$ et 55.

(79) Accord cadre sur le règlement de la question nucléaire entre la Corée du Nord et les États-Unis, Documents d'actualité internationale, $1^{\mathrm{er}}$ janvier $1995, \S 3$. 
ex-Républiques soviétiques d'autre part sont rédigés en termes identiques et comportent à la fois des garanties positives et négatives (80). Les mémorandums dont il est question ici constituent des engagements communs des trois puissances nucléaires à fournir des garanties de sécurité séparément à la Bélarus, au Kazakhstan et à l'Ukraine. En dépit d'un certain formalisme juridique, ils ne font que reprendre le contenu des déclarations unilatérales énoncées par ces puissances en 1994 et constituent la répétition d'engagements formulés dans le passé en faveur des ENDAN parties au TNP.

Certains États comme l'Ukraine (81) ont estimé que ces mémorandums pourraient servir de base à l'élaboration d'un accord universel juridiquement contraignant sur les garanties de sécurité. Mais, en dépit de son originalité, cette solution ne semble pas soulever l'enthousiasme des PNA en raison notamment des insuffisances évoquées précédemment. Ces derniers sont plutôt favorables à l'idée d'adopter une formule commune à inclure dans une convention internationale et visant à garantir sans conditions tous les ENDAN contre l'emploi ou la menace de l'emploi des armes nucléaires. Cette proposition se traduit chaque année depuis 1978 par une résolution votée par l'Assemblée générale (82). Depuis 1989, cette résolution est adoptée sans aucun vote négatif, les trois puissances occidentales préfèrent désormais s'abstenir, rejoints en cela par la Russie tandis que la Chine (83) et les PNA appuient le texte. La résolution se réjouit "qu'il n'y a à la Conférence du désarmement aucune objection de principe à l'idée d'une convention internationale visant à garantir les États non dotés d'armes nucléaires contre l'emploi ou la menace de ces armes»(84). Si, en effet aucun ÉDAN ne s'oppose officiellement à l'idée d'une convention internationale, les trois puissances occidentales restent sceptiques sur sa faisabilité comme l'illustre leur insistance sur l'importance et la crédibilité des engagements unilatéraux qu'elles ont formulés. Il s'agit ici d'élaborer un accord international au sens de l'article 2 de la Convention de Vienne de 1969. Comment envisager cet accord?

L'une des principales difficultés sur la voie d'une convention réside dans l'identification des États chargés de négocier son élaboration. En d'autre termes, la négociation serait-elle engagée entre les puissances nucléaires uniquement ou impliquerait-elle tous les États? Dans la mesures ou l'engagement de non-emploi d'armes nucléaires ne peut être donné que par les EDAN, la contribution des États garants est fondamentale. Mais, la négociation nécessite également la participation des États bénéficiaires des garanties, ces derniers s'engageant en échange à garder leur statut non-nucléaire. A ce propos, les PNA ont toujours insisté pour que la convention résulte d'une négociation entre tous les membres de la Conférence du désarmement, organe où ils sont largement représentés. Il est évident que

(80) Voir $\mathrm{CD} / 1285, \mathrm{CD} / 1286$ et $\mathrm{CD} / 1287$.

(81) S/PV.3514.

(82) La question des garanties négatives de sécurité figurait dès 1978 dans deux points de l'ordre du jour des sessions de l'Assemblée générale inscrits à l'initiative de l'URSS et du Pakistan et faisant l'objet de deux résolutions. Depuis 1979 et ce jusque en 1990, la Bulgarie a pris le relais de l'URSS. Depuis 1990, l'Assemblée n'adopte qu'une seule résolution au titre de la question sur la base d'un projet du Pakistan (voir A/52/598 du 19 novembre 1997 et A/C.1/52/L.41).

(83) La Chine soutient officiellement cette revendication mais semble accorder sa préférence à un accord sur le non-emploi en premier, CD/1281, p. 145.

(84) Conclusion d'arrangements internationaux efficaces pour garantir les États non dotés d'armes nucléaires contre l'emploi ou la menace de ses armes, résolution 52/36 adoptée par 116 voix contre 0 et 51 abstentions ( 9 décembre 1997). 
la présence des ENDAN en tant que partie prenante à la négociation est une assurance que leurs préoccupations seront prises en compte.

Comment se présenterait formellement l'instrument souhaité ? A ce propos, deux cas de figures peuvent exister : un accord de type multilatéral ou de type plurilatéral. On retrouve ici la distinction qu'on établit souvent entre "traité ouvert" et "traité fermé". Un instrument multilatéral serait ouvert à tous les États quel que soit leur statut nucléaire. Le TNP pourrait être un modèle puisqu'il associe des droits et obligations spécifiques aux EDAN (articles $1^{\mathrm{er}}, 3 \S 2$, et 6 ), mais aussi aux ENDAN (articles $2,3 \S 1$ et 4,4 $\S 1,5 \S 2$, ainsi que 7 ) et des engagements communs (notamment art. $4 \S 2$, $5 \S 1$ ). Dans cet accord multilatéral, on préciserait dans le dispositif deux types d'obligations : ceux des ÉDAN de ne pas employer ou menacer d'employer d'armes nucléaires contre les ENDAN parties et ceux des ENDAN de respecter un statut d'absence d'armes nucléaires. L'accord multilatéral suppose une négociation impliquant tous les États, même si celle-ci était menée essentiellement au sein de la Conférence du désarmement, un organe restreint mais ou les différentes sensibilités politiques et une répartition géographique équitable permettent de relativiser son caractère non universel.

On pourrait aussi imaginer un accord de forme plurilatérale, c'est à dire qu'il ne serait ouvert qu'aux seuls États dotés de l'arme nucléaire. La négociation de cet instrument exclut à priori les autres États, mais ces derniers pourraient être liés à travers un protocole où ils prendraient note des garanties négatives formulées dans l'accord principal tout en réaffirmant leur statut non-nucléaire. Il s'agit ici d'un modèle juridique qui a déjà fait ses preuves dans le cadre du mécanisme instituant les ZEAN qui comporte un double niveau d'engagements définis dans le traité principal pour les États parties à la zone et dans un protocole pour les puissances nucléaires.

L'efficacité d'une convention sur les garanties de sécurité est fonction de son caractère universel, c'est à dire que tous les États y soient associés. Le meilleur moyen d'y parvenir est de permettre aux États parties et en particulier aux puissances nucléaires qui sont ici les plus sollicitées, d'assortir la signature et la ratification dudit instrument de déclarations interprétatives qui refléteraient leurs préoccupations de sécurité. Ainsi, le projet pakistanais de Convention internationale sur les garanties négatives contient une disposition stipulant que l'engagement de non-emploi des ÉDAN «est pris sans préjudice des obligations des États parties à la présente Convention découlant des traités portant création des zones exemptes d'armes nucléaires»(85). Cette précision signifierait que les conditions d'octroi de garanties négatives formulées au moment de la signature des protocoles aux traités de ZEAN pourraient être reprises au moment de l'adhésion à la future convention. On peut également inclure dans l'arrangement international une clause de retrait permettant à un ÉDAN de renoncer à son engagement dans le cas ou un ENDAN ne remplirait plus les critères de qualification retenus dans la formule commune.

(85) Article $1^{\text {er }} \S 2$ du projet de Convention internationale sur les garanties aux États non-nucléaires contre l'utilisation ou la menace d'utilisation d'armes nucléaires, A/C.1/33/L.15. Le Nigeria s'est aussi déclaré favorable à une telle disposition (NPT/CONF.IV/11, p. 10). 


\section{Critères de qualification des États bénéficiaires}

Les États susceptibles de recevoir les garanties négatives de sécurité sont les États non-dotés de l'arme nucléaire, c'est-à-dire ceux qui n'appartiennent pas à la catégorie visée dans l'article IX $\S 3$ du TNP (Chine, États-Unis, France, Royaume-Uni et Russie) (86). Pourtant, la définition du terme "État non doté d'arme nucléaire" peut soulever quelques difficultés.

Pourrait-on considérer aujourd'hui l'Inde et le Pakistan qui ont fait exploser des engins nucléaires comme des États non nucléaires? Et que penser d'Israël qui disposerait déjà de la capacité nucléaire? Les États dits du «seuil» (Threshold countries) (87) sont-ils considérés comme des ENDAN au motif qu'ils n'auraient jamais officiellement reconnu leur statut nucléaire? Ce problème de définition fut abordé au sein du Comité spécial de la Conférence du désarmement qui a alors rappelé que pour la majorité des délégations "le terme d'«État non doté d'armes nucléaires" ne présentait en soi aucune ambiguïté; il s'expliquait de lui-même et, cela étant, il rendait inutile tout développement sur le statut de ces États". (88) Un ENDAN est donc un État qui a renoncé à mettre au point et à acquérir l'arme nucléaire, cet engagement est assumé dans un instrument international juridiquement contraignant. Le statut non-nucléaire d'un État est aujourd'hui consacré par deux types d'instruments internationaux : le TNP et les accords instituant les ZEAN.

Les États parties à ces instruments sont donc naturellement qualifiés pour bénéficier de garanties négatives aussi bien que positives étant entendu qu'ils doivent respecter ce statut. Le Royaume-Uni avait en effet jugé nécessaire de préciser dans sa déclaration unilatérale de 1995 qu'il retirerait ses garanties de non-emploi "en cas de violation substantielle, par tel ou tel bénéficiaire, des obligations de non-prolifération qui incombent à celui-ci au titre du traité sur la non-prolifération des armes nucléaires" (89). Le cas de l'Irak, État partie au TNP mais qui a développé un programme nucléaire lui permettant à terme de se doter de la bombe pourrait expliquer cette précision. Elle implique donc que le statut non-nucléaire de l'État bénéficiaire des garanties doit être vérifiable. L'AIEA est l'organisation internationale qualifiée en vertu de ses statuts mais également de l'article III du TNP pour mener des missions de contrôle sur les activités nucléaires des ENDAN (90).

On a aussi proposé que les garanties négatives ne pourraient être fournies qu'aux ENDAN qui n'appartiendraient pas à une alliance militaire où participerait une puissance nucléaire et qui n'accueilleraient pas d'armes nucléaires sur leur territoire pour le compte d'un ÉDAN. Les projets de Convention sur le non-recours à l'arme nucléaire contre les ENDAN proposés en 1978 par l'URSS et le Pakistan vont dans ce sens. L'article premier du projet soviétique demandait que les ÉDAN «s'engagent à ne pas utiliser ou

(86) Cet article stipule qu'“au fins du présent traité un État équipé d'armes nucléaires est celui qui a fabriqué et a fait exploser une arme nucléaire ou un autre dispositif nucléaire explosif avant le $1^{\text {er }}$ janvier 1967 ".

(87) L'Afrique du Sud a démantelé son programme nucléaire militaire sous contrôle de l'AIEA à partir de 1993. Voir StumpF, Waldo, South Africa's Nuclear Weapons. Program : from Deterrence to Dismantlement, Arms Control Today, Vol. 25, n' 10, December 1995/January 1996, pp. 3-8.

(88) $\mathrm{CD} / 825$, p. 213.

(89) $\mathrm{CD} / 1306$, p. 2.

(90) Tenant compte de l'expérience irakienne, le système de garantie anti-prolifération de l'Agence a été renforcé en mai 1997 dans un sens plus intrusif (document INFICIRC/540). 
menacer d'utiliser des armes nucléaires contre les États non nucléaires parties à la présente convention qui renoncent à fabriquer et à acquérir des armes nucléaires et ne possèdent pas d'armes nucléaires sur leur territoire ou dans quelque zone que se soit se trouvant sous leur juridiction et leur contrôle, sur terre, en mer, dans l'atmosphère ou dans l'espace extra-atmosphérique» (91). Quant à l'article premier du projet pakistanais, il stipule que les ÉDAN «s'engagent à ne pas utiliser ou à ne pas menacer d'utiliser des armes nucléaires contre les États non dotés d'armes nucléaires qui ne sont pas parties aux arrangements relatifs à la sécurité nucléaire conclus par certains États dotés d'armes nucléaires» (92).

Le point commun à ces deux textes réside dans la référence à une convention internationale sur les garanties négatives. Mais des différences apparaissent lorsqu'il s'agit d'identifier plus précisément les bénéficiaires. Alors que le projet soviétique écarte des garanties les ENDAN qui accueillent des armes nucléaires sur leur territoire (critère de non-stationnement) visant implicitement la présence en Europe d'armes nucléaires sous commandement de l'OTAN, l'objectif du Pakistan est de n'accorder ces garanties qu'aux ENDAN qui ne seraient pas liés à une puissance nucléaire par des accords de sécurité afin de faciliter l'adoption de garanties négatives en faveur des États qui ne bénéficient d'aucun parapluie nucléaire. En fait, les deux projets excluent des garanties les mêmes États. En effet les ENDAN ayant sur leur territoire des armes nucléaires appartenant à une grande puissance sont également liés à ladite puissance par des arrangements de sécurité nucléaires collectifs (ex : États membres de OTAN) ou bilatérales (ex : États-Unis/Japon) (93). L'argument que l'on pourrait avancer ici est qu'un ENDAN appartenant à une alliance militaire au côté de puissances nucléaires bénéficie déjà d'arrangements de sécurité avec ladite puissance. Il est clair que la présence d'armes nucléaire sur son territoire l'exposerait en cas de conflit à une attaque nucléaire. Il pourrait tout au plus bénéficier d'un engagement de non-emploi en premier. De surcroît, en tant qu'allié d'une puissance nucléaire, il est couvert par la dissuasion en cas d'agression comme l'illustre la clause de non-attaque contenue dans les déclarations formulées par quatre des cinq puissances nucléaires.

Les deux projets de conventions ont été jugés inacceptables par les puissances nucléaires occidentales. Le Nigeria a proposé une alternative intéressante, l'approche par la "catégorisation". Elle consiste à classer les ENDAN en trois catégories, selon leur situation en matière de sécurité : les États ne possédant pas d'armes nucléaires et n'appartenant pas à une alliance militaire avec un ÉDAN; ceux appartenant à une alliance militaire avec un ÉDAN mais qui n'ont pas d'armes nucléaires implantées sur leur territoire; ceux enfin appartenant à une alliance militaire et qui ont des armes nucléaires d'un autre État implantées sur leur territoire. S’il est

(91) Convention internationale sur le renforcement des garanties de sécurité des États nonnucléaires, A/C.1/33/L.6. Il est surprenant que ce projet mentionne le non stationnement dans l'espace extra-atmosphérique, espace qui non seulement échappe à la souveraineté des États mais est déjà soumis à un régime de dénucléarisation conformément à l'article 4 du Traité sur l'espace extra-atmosphérique du 27 janvier 1967.

(92) Convention internationale sur les garanties aux États non-nucléaires contre l'utilisation ou la menace d'utilisation d'armes nucléaires, A/C.1/33/L.15.

(93) Cependant, il faut remarquer que les ENDAN parties à des arrangements de sécurité avec des ÉDAN ne possèdent pas tous des armes nucléaires sur leur territoire (ex : États-Unis/Arabie-Saoudite). 
demandé aux puissances nucléaires de s'engager à ne pas menacer ou employer d'armes nucléaires contre tout les ENDAN, les engagements que donneraient ces derniers en contrepartie, seraient modulés selon la catégorie à laquelle ils se rattachent. Ainsi, les ENDAN relevant du premier groupe respecteraient simplement la renonciation à mettre au point et à acquérir des armes nucléaires. Ceux appartenant à la deuxième catégorie prendraient le même engagement mais s'abstiendraient en outre de contribuer à toute attaque contre un État partie à l'accord sauf en cas de légitime défense. Enfin, le dernier groupe d'ENDAN devrait en plus des obligations assumées par les autres ENDAN s'engager sous "une forme qui soit satisfaisante" pour les puissances nucléaires à ce que les armes nucléaires stationnées sur leur territoire ne soient pas utilisées contre tout autre État sauf en cas de légitime défense (94).

Cette proposition fut examinée à l'occasion d'un échange de vue au sein du Comité spécial, bien qu'elle n'a pas fait l'objet jusqu'ici de discussions approfondies (95). Elle a le mérite de reposer sur un équilibre dans les engagements des ÉDAN et des ENDAN et de prendre en considération la diversité de situations sur le plan militaire. Sa mise en œuvre pourrait toutefois soulever des difficultés. En effet, comment intégrer dans un instrument international unique les obligations assumées par quatre catégories d'États (les ÉDAN et les trois groupes d'ENDAN)? On constate que le première catégorie d'ENDAN n'est pas soumise au critère de non-attaque appliqué aux deux autres. L'engagement de non-attaque contre «tout État partie" assumé par les ENDAN appartenant aux derniers groupes mérite une explication. S'appliquerait-il stricto sensu ou ne concerne-t-il que les attaques menées en alliance avec une puissance nucléaire? Qu'est-ce que on entend par "une forme qui soit satisfaisante" à propos de l'engagement que prendraient les ENDAN afin que les armes nucléaires stationnées sur leur territoire ne soient pas utilisées contre tout autre État sauf en cas de légitime défense? Cet engagement serait-il négocié avec la puissance concernée ou nécessiterait-il l'accord de toutes les puissances nucléaires?

Cette proposition illustre à bien des égards les difficultés réelles auxquelles tout effort visant à élaborer des garanties négatives efficaces se heurtent (96). Il reste, qu'elle n'en constitue pas moins une base de travail pour clarifier les engagements réciproques des États garants et des États qualifiés à énoncer dans la formule commune, et pour, en fin de compte, sortir de l'impasse actuelle..

Ainsi, de sérieuses divergences subsistent entre les États sur la définition d'une formule commune visant à garantir les ENDAN contre l'emploi et la menace d'emploi de l'arme nucléaire. Le Comité spécial de la Conférence du désarmement n'a pu à ce jour mener des négociations de fond sur un arrangement international efficace. Il a limité ses travaux à des consultations officieuses au cours desquelles les délégations se sont contentées de réaffirmer leur positions respectives. La proposition du Président du Comité tendant à constituer un groupe de rédaction chargé de soumettre des

(94) Conclusion immédiate d'arrangements internationaux efficaces en vue de garantir les ENDAN contre la menace ou l'emploi d'armes nucléaires, CD/768.

(95) $\mathrm{CD} / 825$, p. $215, \S 10$.

(96) Pour une présentation plus exhaustive des propositions, voir Thomas BernaUer, Nuclear Issues on the Agenda of the Conference on Disarmament, (UNIDIR: Genève), Publications des Nations Unies (New York), 1991, pp. 1-28. 
propositions concrètes n'a pu être adoptée faute de consensus (97). Le Comité spécial a dû consacrer une grande partie de sa session de 1994 - il n'a pu se réunir depuis - à un "échange de vues informel mais approfondi" sur la base d'un questionnaire établi par son Président (98). Aucun progrès significatif n'a été enregistré sur les questions de fond abordées dans ce questionnaire, peu d'États ayant même jugé utile à ce stade d'y répondre. Le comité spécial a avoué son échec en reconnaissant "que la complexité des questions en jeu continuait d'empêcher la conclusion d'un accord sur une formule commune»(99).

\section{Conclusion}

En dépit du soutien dont elle bénéficie aux Nations Unies, l'adoption d'arrangements internationaux efficaces pour garantir les ENDAN contre la menace ou l'emploi d'armes nucléaires se révèle être une entreprise complexe. Les propositions visant à limiter l'emploi de l'arme nucléaire soulèvent en effet des problèmes sensibles liés aux perceptions de sécurité des États, et en particulier à l'attachement des puissances nucléaires aux doctrines de dissuasion (100). Avec la disparition de la rivalité Est/Ouest qui a créé un contexte international favorable, on aurait pu espérer un progrès significatif en matière de limitation de l'emploi de l'arme nucléaire. En fait, la prudence des ÉDAN en matière d'octroi de garanties de sécurité est de règle, mais cette position négative est de plus en plus intenable face aux pressions croissantes dont elles font l'objet. L'impatience de l'Assemblée générale des Nations Unies qui examine la question depuis vingt ans transparaît dans les formules aussi pressantes les unes que les autres appelant les États à "s'entendre rapidement", à "travailler activement" et à "redoubler d'efforts" en vue "de conclure des arrangements internationaux efficaces pour garantir les Etats non dotés d'armes nucléaires contre l'emploi ou la menace de ces armes"(101). Cet appel s'adresse en particulier aux puissances nucléaires que les PNA accusent de ne pas être à la hauteur de la responsabilité spéciale qui est la leur, pour sortir la question de l'impasse dans laquelle elle se trouve depuis vingt ans (102).

Espérons que la nouvelle donne nucléaire en Asie du Sud, si elle porte un sérieux coup à l'ordre nucléaire mondial favorable aux cinq grandes puissances aura au moins le mérite de relancer les discussions sur les garanties de sécurité à apporter aux ENDAN ne serait-ce que pour les dissuader de suivre l'exemple indien et pakistanais. Aujourd'hui, il ne fait aucun doute que l'inexistance d'assurances de sécurité universelles et crédi-

(97) Rapport du Comité spécial sur des arrangements internationaux efficaces pour garantir les États non dotés d'armes nucléaires contre l'emploi ou la menace de l'emploi de ces armes, $\mathrm{CD} / 1275$, p. $2, \S 5$ et 7 .

(98) Le questionnaire portait sur les aspects suivants : les perceptions des menaces et en particulier de la menace nucléaire; les dangers potentiels pour la sécurité nationale et internationale; les bénéficiaires et les fournisseurs de garanties; la portée et le type de sanctions dans un système de sécurité collective, y compris un rôle possible pour l'ONU; le cadre d'un accord éventuel, document CD/SA/CRP.25.

(99) CD/1173, pp. 83-85

(100) Les PNA estiment qu'avec la fin de la Guerre froide, "les forces de dissuasion ont perdu toute pertinence ", Déclaration du Sommet du Mouvement des non-alignés réuni à Carthagène du 18 au 20 octobre 1995, A/50/752 - S/1995/1035 Annexe III, $\S 82$.

(101) Résolution $52 / 36$, § 1 et 3 à 5 .

(102) $\mathrm{CD} / 825$, p. 221. 
bles prohibant l'emploi d'armes nucléaires contre les États qui en sont dépourvus ajoutée à l'absence d'un progrès significatif vers le désarmement nucléaire constituent un dangereux ingrédient qui risque de faire imploser le régime international de non-prolifération. Puissent les explosions du Rajasthan et du Balouchistan produire l'onde de choc salutaire à la prise de conscience universelle face à la menace nucléaire.

Mais les arrangements de sécurité anti-nucléaires ne sont pas une fin en soi et ne peuvent être isolés du contexte dans lequel ils sont accordés. Ils s'insèrent dans un système de mesures visant à renforcer la non-prolifération des armes nucléaires lequel système n'est crédible que s'il implique le désarmement nucléaire. Dès lors, une interdiction de l'emploi de l'arme nucléaire contre les ENDAN, sous quelque formule que se soit, restera fragile tant que cette arme existera. En définitive, la meilleure garantie contre l'emploi ou la menace de l'emploi de l'arme nucléaire pour ces pays et pour la communauté internationale dans son ensemble réside dans un processus de désarmement visant à terme l'élimination de cette catégorie d'arme de destruction massive.

N.B. : Au moment où nous mettons sous presse nous apprenons que la Conférence du désarmement a pu aboutir à un accord sur la constitution d'un comité ad hoc sur les garanties négatives de sécurité avec un mandat de négociation. Mais, la persistance des positions des principaux Etats au cours de la session 1998 de la Conférence laisse peu d'espoir quant à l'issue rapide de cette négociation. 